\title{
Proton acceleration in a single-loop disrupted during collision of two moving solitary magnetic kinks
}

\author{
J. I. Sakai and A. Kakimoto
}

\author{
Laboratory for Plasma Astrophysics, Faculty of Engineering, Toyama University, 3190, Gofuku, Toyama, 930-8555, Japan \\ e-mail: sakaijun@eng.toyama-u.ac.jp
}

Received 17 March 2004 / Accepted 9 June 2004

\begin{abstract}
We investigate a new model of single-loop flare based on the fact that a magnetic flux tube with axial current can be explosively disrupted during collision of two moving solitary magnetic kinks (MoSMaK), particularly paying attention to an acceleration mechanism of high energy protons. By using three-dimensional electromagnetic fields obtained from a resistive three-dimensional MHD equations during the single-loop flare, we investigate the orbit of many protons to obtain their energy spectra. We found that the protons can be accelerated to $\gamma$-ray-emitting energies $(>1 \mathrm{MeV})$ with double power-law spectra up to about $25 \mathrm{MeV}$. There appears a breaking point of the index of the power-law spectra from 1.8 to 2.1 near $10 \mathrm{MeV}$. The protons are accelerated mainly in one direction along the loop by the electric field produced near the three-dimensional localized current associated with the magnetic reconnection process in the disrupted loop. We study the loop disruption for two different plasma beta in the center of the loop; $\beta=0.5$ and $\beta=0.05$. In a high beta case $(\beta=0.5)$, the disruption of the loop is more violent than that of $\beta=0.05$. We found that the proton acceleration for the case of $\beta=0.5$ occurs mainly at two different regions; one is inside the loop and the other is in the upper part of the disrupting loop. However, the energy spectra of protons are very similar for both cases.
\end{abstract}

Key words. acceleration of particles - magnetohydrodynamics (MHD) - plasmas - Sun: flares

\section{Introduction}

When energetic protons accelerated during impulsive flares collide with solar atmosphere, they produce excited nuclei, which emit prompt nuclear de-excitation lines, as well as secondary neutrons and positrons that results in the delayed 2.223 MeV neutron-capture and $511 \mathrm{KeV}$ positron-annihilation line emission. In most strong flare events the time profile of the prompt gamma-ray line emission is observed to be very similar to that of the bremsstrahlung hard X-rays emitted by energetic electrons. This suggests that the acceleration and propagation of the flare-accelerated protons and electrons are closely related. The most typical event among them is the 1980 June 7 flare observed by the SMM (Forrest \& Chupp 1983), which flare was explained by the current loop coalescence model (Tajima et al. 1982; Sakai \& Ohsawa 1987; Sakai \& De Jager 1996; Aschwanden 2002). Sakai \& de Jager (1996) gave a review of the high-resolution observations of solar plasma loops with simulations of current-carrying loops and tried to arrive at the understanding of solar flare phenomena. In Chapter 7 in their review, they summarized the understanding of highenergy particle acceleration processes during and after solar flares. There are two types of solar flares: Confined/Impulsive and Eruptive/Dynamic flares. Confined/Impulsive flares are spatially compact and as a fairly general rule they show impulsive hard X-ray and/or microwave bursts. Eruptive/Dynamic flares, on the other hand, are spatially more extended and last longer. As proposed by de Jager (1988) and Sakai \& de Jager (1991), there are three phases of acceleration in a fully developed eruptive/dynamic flare. The relevant observations regarding the first two phases are:

(1) the acceleration times of electrons and protons to energies of the order of one $\mathrm{MeV}$ are of the order of a second.

(2) $\mathrm{MeV}$ protons are accelerated nearly simultaneously with $\mathrm{MeV}$ electrons.

Many theoretical works (Forman et al. 1986; Simnett 1995; Sakai \& de Jager 1996; Miller et al. 1997; Aschwanden 2002) to explain the above observational requirements have been done.

However, the location, size and geometry of the accelerated proton collision region remains unknown until now. Recent paper by Hurford et al. (2003) presents the first gammaray images of a solar flare taken from the Reuven Ramaty High Energy Solar Spectroscopic Imager (RHESSI) for the X4.8 flare of 2002 July 23. The result shows that the centroid of the $2.223 \mathrm{MeV}$ image was found to be displaced by $20 \pm 6$ arcsec from that of the $0.3-0.5 \mathrm{MeV}$, implying a difference in acceleration and/or propagation between the accelerated electron and proton populations near the Sun. The fact that proton-associated gamma-ray source does not coincide with the electron-bremsstrahlung sources suggests that the protons 
would be accelerated in one direction by the DC electric field and could subsequently interact in spatially separated sources. Therefore it is now important to investigate in details the proton acceleration processes for different types of flares.

Among Confined/impulsive flares single-loop flares occur in a single loop. We consider single-loop flares an elementary case of the class of confined/impulsive flares. Alfvén \& Calqvist (1967), Spicer (1977), Colgate (1978), Uchida \& Shibata (1988), Zaitsev et al. (2000) proposed the flare energy release in the frame of a simple loop. These models are divided into two classes: (1) electric current dissipation models due to micro-instabilities and (2) magnetic reconnection models in a current loop. Recently Uchida et al. (2001) and Miyagoshi et al. (2001) proposed that loop flares may be due to the transport of energy and mass by torsional Alfvén waves, when two torsional Alfvén wave packets collide high in a coronal loop. Their model is based on the observational evidence that before a loop flare brightened the footpoints brightened first, then the bright region moved up to the loop top.

Even though there are no clear observational evidences showing the precursor phase of a solar flare, recent observation (Harra et al. 2001) showed nonthermal velocity increase about ten minutes before the onset of a flare. Recent observations (Chae et al. 1999, 2000; Lee et al. 2000) also showed that many flux tubes are in dynamical state with intermittent plasma flows along the flux tubes. The magnetic fibrils also have plasma flows confined in the flux tubes. Therefore we focus on the dynamics of magnetic flux tubes with plasma flows along the tubes. The origin of intermittent plasma flows in the flux tubes is not clear from the observations, but there are a few theoretical predictions to drive plasma flows along the small scale flux tubes.

To understand the dynamics of small-scale magnetic flux tubes with a diameter of about $100 \mathrm{~km}$ in the supergranular network, Furusawa \& Sakai (2000) investigated the collision process of two flux tubes and they showed that shock waves can be excited from a region where two magnetic flux tubes with weak electric current collide with each other. They also showed that plasma up-flows are generated along the flux tubes, when two magnetic flux tubes collide with X-type configuration. The shock waves may in turn collide with other flux tubes. Sakai et al. (2000) studied the interaction process between a magnetic flux tube with both weak current and strong current, and shock waves. They found that when the shock wave collides with a magnetic flux tube with weak electric current, surface Alfvén waves can be generated and propagate along the flux tube. However, when the shock wave collides with a magnetic flux tube with strong current, body Alfvén waves can be generated and propagate along the flux tube. It was also shown that when we take into account of the effect of background density inhomogeneity due to gravity, there occurs a strong upward plasma jet along the flux tube as well as the surface Alfvén waves.

Recently Sakai et al. (2002) investigated head-on and rearend collision process of dense plasma blobs moving along a magnetic flux tube with axial current, by using a resistive 3D MHD code. They found a new nonlinear elementary excitation called "moving solitary magnetic kink" (MoSMaK) that can be excited near the interface of colliding plasma blobs. The MoSMaK is characterized with an isolated magnetic flux ring and a pair of counter-rotating vortex rings. They studied the collision process of two MoSMaKs along a straight magnetic flux tube. It was shown that a twisted magnetic flux tube can be explosively disrupted during the collision of two MoSMaK's, resulting in strong emission of magnetosonic waves. They also investigated the interaction process of two current loops with the MoSMaKs. The two loop interaction can be enhanced through the collision process of the MoSMaK. Sakai et al. (2003) also investigated rear-end collision process of dense plasma blobs moving along a magnetic flux tube with very weak axial current, by using a resistive 3-D MHD code. They found another new nonlinear elementary excitation called "moving solitary magnetic sausage" (MoSMaS). They proposed a new mechanism of formation of sporadic plasma jet from a disrupting magnetic flux tube during collision of two MoSMaSs.

Sakai (1990) showed that, during 3D X-type current loop coalescence and under suitable assumptions of the size and other physical parameters in the region of acceleration, protons and electrons may be accelerated promptly (i.e., within less than $1 \mathrm{~s}$ ) to $\approx 100 \mathrm{GeV}$ and $\approx 100 \mathrm{MeV}$, respectively. De Jager \& Sakai (1991) showed that the duration of impulsive phase bursts (5-25 s) observed during the impulsive phases of flares can be explained quantitatively by the mechanism of X-type current-loop coalescence. Sakai (1992) developed a model for long-duration gamma-ray/proton flares (the "gradual GR/P flares") in order to explain prompt proton and electron acceleration during the impulsive phase. He determined the electric fields during the implosion phase of the current sheet from the MHD equations, and investigated the motion of test protons and electrons. Results showed that, under reasonable assumptions of the size and velocities in the reconnection area, both protons and electrons can be accelerated promptly within $1 \mathrm{~s}$ to $\sim 70 \mathrm{MeV}$ and $\sim 200 \mathrm{MeV}$, respectively. However, the above previous works did not give the energy spectrum of accelerated particles. In the first phase of acceleration, current loop coalescence is one of the most plausible models of solar flares. Although triggering mechanisms of most of complex flares are still puzzling so far, several examples of solar flares triggered by current loop coalescence were observed by Yohkoh (see for the review, Sakai \& de Jager 1996). In the case of partial reconnection of kink-unstable loops coalescence (Nishikawa et al. 1994), it seems that electrons can be promptly accelerated to relativistic energies, while the problem of proton acceleration has not been investigated because of limitation of full proton dynamics by using a particle simulation model.

Bulanov (1980) first showed the energy spectrum of accelerated particles in the simple X-type configuration near the reconnection region, which is given by $f(E) \sim \operatorname{Exp}\left(-\left(E / E_{0}\right)^{3 / 4}\right)$ for $E \ll m c^{2}$. Deeg et al. (1991) investigated the same problem by using numerical simulation. Their results were in good agreement with the theory by Bulanov (1980). Nocera et al. (1996) investigated chaotic behavior of a particle motion in the same field configuration.

Mori et al. (1998) investigated the behavior of protons near an X-type magnetic reconnection region by numerical 
simulations. The magnetic field is taken to be hyperbolic and time stationary with a uniform electric field perpendicular to the magnetic field. They also studied the effects of the magnetic field along the uniform electric field. They found from many parametric runs that the energy spectrum of accelerated protons near an X-type magnetic reconnection region is universal with a power-law spectrum $E^{-\gamma}$, where the power-law index $\gamma$ is about 2.0-2.2. The acceleration time of protons with the energy range of $1-20 \mathrm{MeV}$ is very rapid and within $\sim 10^{2} \omega_{\mathrm{ci}}^{-1}$ which is much less than $1 \mathrm{~s}$ for solar flare plasmas. Heerikhuisen et al. (2002) and Craig et al. (2002) investigated the proton dynamics under a self-consistent MHD reconnection solution and found that the proton energy spectra approximates a power law $E^{-1.5}$ nonrelativistically, but steepens slightly at the higher energies. Hamilton et al. (2003) studied the proton energy spectra under the model fields obtained from solutions of the linearized MHD equations for reconnecting region. They showed that some cases the energy distributions exhibit a bump-on-tail in the $\mathrm{MeV}$ range.

In this paper we investigate a new model of single-loop flare based on the fact that a magnetic flux tube with axial current can be explosively disrupted during collision of two moving solitary magnetic kinks (MoSMaK), particularly paying attention to an acceleration mechanism of high energy protons. By using three-dimensional electromagnetic fields obtained from a resistive three-dimensional MHD equations during the singleloop flare, we investigate the orbit of many protons to obtain their energy spectra. We found that the protons can be accelerated to $\gamma$-ray-emitting energies $(>1 \mathrm{MeV})$ with double power-law spectra. The protons are accelerated mainly in one direction along the loop by the electric field produced near the three-dimensional localized current associated with the magnetic reconnection process in the disrupted loop. We study the loop disruption for two different plasma beta in the center of the loop; $\beta=0.5$ and $\beta=0.05$. In a high beta case $(\beta=0.5)$, the disruption of the loop is more violent than that of $\beta=0.05$. We found that the proton acceleration for the case of $\beta=0.5 \mathrm{oc}-$ curs mainly at two different regions; one is inside the loop and the other is in the upper part of the disrupting loop. However, the energy spectra of protons are very similar for both cases.

In Sect. 2 we present our methods to obtain the proton energy spectra during the single-loop flare. In Sect. 3 we briefly review our model of the single-loop flare during collision of two MoSMaKs obtained from a resistive MHD equation. In Sect. 4 we investigate many proton orbits under the electromagnetic fields during the collision of two MoSMaKs to find their energy spectra. In final section we summarize our results.

\section{Method of simulation}

In this section we describe two methods of simulation to obtain the energy spectra of the protons accelerated in a singleloop disrupted during collision of two MoSMaks. Firstly we present briefly a model of a single-loop flare by using a threedimensional resistive MHD simulation to obtain the electromagnetic fields during the disruption of the loop. Next we present the simulation method to obtain the orbits of many protons under the electromagnetic fields obtained from the
MHD simulation. The validity of the second test proton simulation implies that nonthermal proton population accelerated under the MHD electromagnetic fields is small compared with the background thermal population sustaining the MHD fields.

\subsection{Resistive $M H D$ simulation}

A resistive 3-D MHD code with recently proposed Artificial Wind (AW) numerical scheme (Sokolov et al. 1999) with splitting over the spatial coordinates is employed. The basis of the AW scheme is the fact that fundamental physical invariance, Galilean (or more general, Lorenz) invariance, allows to solve the governing equation in different steadily moving frame. The principle of the AW scheme is that the frame of reference may be chosen in such a way that the flow under simulation is supersonic there. The problem of upwinding becomes trivial and considerably facilitated versions of discontinuity-capturing schemes may be employed. An extra velocity (Artificial Wind) is added to the velocity of the flow under simulation when the system of coordinates is changed.

The MHD equations are numerically solved in a conservative form as follows:

$\frac{\partial \rho}{\partial t}+\frac{\partial}{\partial x_{i}}\left(\rho V_{i}\right)=0$

$\frac{\partial\left(\rho V_{i}\right)}{\partial t}+\frac{\partial}{\partial x_{j}}\left[\rho V_{i} V_{j}+\left(p+B^{2}\right) \delta_{i j}-2 B_{i} B_{j}\right]=0$

$\frac{\partial B_{i}}{\partial t}+\frac{\partial}{\partial x_{j}}\left(V_{j} B_{i}-V_{i} B_{j}\right)=\frac{1}{R_{\mathrm{m}}} \frac{\partial^{2} B_{i}}{\partial x_{j}^{2}}$

$$
\begin{aligned}
& \frac{\partial}{\partial t}\left(\frac{\rho V^{2}}{2}+\frac{p}{\gamma-1}+B^{2}\right)+ \\
& \frac{\partial}{\partial x_{i}}\left[V_{i}\left(\frac{\rho V^{2}}{2}+\frac{\gamma p}{\gamma-1}+2 B^{2}\right)-2 B_{i} B_{j} V_{j}+q_{i}\right]=0
\end{aligned}
$$

where, $\rho, V_{i}, p$ and $B_{i}$ are the density, velocity, pressure, and magnetic field, $\gamma$ is the adiabatic constant, which is taken to be $\gamma=5 / 3, R_{\mathrm{m}}$ is the magnetic Reynolds number, $\delta_{i j}$ is a unity tensor and $q_{i}$ is a dissipative energy flux. The density, pressure, velocity and magnetic field are normalized by $\rho_{0}, p_{0}, \sqrt{p_{0} / \rho_{0}}$ and $B_{0}=\sqrt{8 \pi p_{0}}$, respectively.

For resistive MHD with a large but finite value of $R_{\mathrm{m}}$ the energy equation Eq. (4) should be obtained as a sum of the equation for plasma energy, in which the Joule heating term is present as follows: $\frac{(\operatorname{crot} \boldsymbol{B})^{2}}{(4 \pi)^{2} \sigma}$ and the equation for magnetic energy which is given by the Eq. (4) multiplied by the magnetic field $B_{i} /(4 \pi)$, all the variables are not normalized here and $\sigma$ is a conductivity. The dissipation of the magnetic field energy is hence given by the term as follows: $\frac{c^{2}}{(4 \pi)^{2} \sigma} B_{i} \Delta B_{i}$. So in the Eq. (4) for the total energy the Joule heating is compensated by the magnetic energy dissipation in a following way:

$\frac{c^{2}}{(4 \pi)^{2} \sigma}\left[B_{i} \Delta B_{i}+(\operatorname{rot} \boldsymbol{B})^{2}\right]=\frac{c^{2}}{(4 \pi)^{2} \sigma} \operatorname{div}[\boldsymbol{B} \times \operatorname{rot} \boldsymbol{B}]$.

So the resistive dissipation in the equation of energy in present only in a form of the additional dissipative energy flux, which, 
in normalized variables, may be written as follows: $q_{i}^{(m)}=$ $R_{\mathrm{m}}^{-1} \frac{\partial}{\partial x_{j}}\left(2 B_{i} B_{j}-B^{2} \delta_{i j}\right)$. The dissipative energy flux due to heat transfer may be also taken into account in a usual form: $q_{i}^{(h)}=$ $\lambda_{i j} \frac{\partial}{\partial x_{j}} \frac{p}{\rho}$. For the sake of simplicity here we fully neglect the non-diagonal part of dissipative energy transfer tensor and substitute all the tensors in $q_{i}$ for unity tensors: $B_{i} B_{j}=B^{2} \delta_{i j} / 3$, $\lambda_{i j}=\lambda_{l l} \delta_{i j} / 3$. The numerical simulation shows that the influence of the dissipative energy flux is insignificant, so we do not try to take it more carefully into account. Finally we admit the dissipative hydrodynamic flux to be as follows:

$q_{i}=\frac{\partial}{\partial x_{i}}\left(-\frac{B^{2}}{3 R_{\mathrm{m}}}-\lambda \frac{p}{\rho}\right)$

The magnetic Reynolds number $R_{\mathrm{m}}=1.3 \times 10^{3}$ and the heat transfer constant $\lambda=2.5 \times 10^{-4}$ are used in simulation. Radiating boundary conditions were used for all directions.

\subsection{Simulation of proton dynamics}

By using the electromagnetic fields obtained from the resistive MHD equations, we investigate many proton orbits to obtain the energy spectra of protons. The normalized relativistic equations of the motion of a proton is given by

$$
\begin{aligned}
\frac{\mathrm{d} \boldsymbol{u}}{\mathrm{d} t} & =\boldsymbol{E}+\frac{\boldsymbol{u} \times \boldsymbol{B}}{\Gamma}, \\
\frac{\mathrm{d} \boldsymbol{x}}{\mathrm{d} t} & =\frac{R \boldsymbol{v}}{\Gamma},
\end{aligned}
$$

where the proton velocity $\boldsymbol{v}=\Gamma^{-1} \boldsymbol{u}$, the electric field $\boldsymbol{E}$, and the magnetic field $\boldsymbol{B}$ are normalized by Alfvén velocity $V_{\mathrm{A}}, E_{0}=$ $V_{\mathrm{A}} B_{0} / c$, and $B_{0}$, respectively. The time is normalized by the proton cyclotron frequency $\omega_{\mathrm{ci}}$, and the length is normalized by the loop diameter $(a)$, respectively. The Lorentz factor $\Gamma$ is given by $\Gamma=\left(1+A^{2} \boldsymbol{u}^{2}\right)^{1 / 2}$, where $A=V_{\mathrm{A}} / c$. The parameter $R$ in Eq. (8) is defined by $R=V_{\mathrm{A}} /\left(\omega_{\mathrm{ci}} a\right)$. In the next simulation we take $A=1 / 300$ and $R=10^{-8}-10^{-6}$.

To advance particle under the guidance of electric and magnetic fields through solving the motion equation numerically, we desire a centered-difference form of the Newton-Lorentz equations of motions (Buneman 1993; Birdsall \& Langdon 1991), where we use the un-normalised equation to make the stability condition clear, namely

$\boldsymbol{u}^{\text {new }}-\boldsymbol{u}^{\text {old }}=\frac{q \delta t}{m}\left[\boldsymbol{E}+\frac{1}{2 \Gamma}\left(\boldsymbol{u}^{\text {new }}+\boldsymbol{u}^{\text {old }}\right) \times \boldsymbol{B}\right]$,

where $q$ is the charge of a particle, $m$ is the mass of a particle, and $\delta t$ is the calculate time step, respectively. As it stands, the equation for $\boldsymbol{u}^{\text {new }}$ is implicit. We choose the method proposed by Boris (1970) to obtain a simpler explicit solution using several steps as the following.

(1) half an electric acceleration: $\boldsymbol{u}_{0}=\boldsymbol{u}^{\text {old }}+q \boldsymbol{E} \delta t / 2 m$,

(2) a pure magnetic rotation:

$$
\boldsymbol{u}_{1}=\boldsymbol{u}_{0}+2 \frac{\boldsymbol{u}_{0}+\boldsymbol{u}_{0} \times \boldsymbol{b}_{0}}{1+b_{0}^{2}} \times \boldsymbol{b}_{0}
$$

where $\boldsymbol{b}_{0}=q \boldsymbol{B} \delta t / 2 m \Gamma$,
(3) another half electric acceleration: $\boldsymbol{u}^{\text {new }}=\boldsymbol{u}_{1}+q \boldsymbol{E} \delta t / 2 m$.

This method produces a rotation through angle

$\theta=2 \arctan \left[\frac{q B}{m} \frac{\delta t}{2}\right]$

The difference of $\theta$ to $\omega_{\mathrm{ci}} \delta t=q B \delta t / m$ is less than one percent error for $\omega_{\mathrm{ci}} \delta t<0.35$. We can obtain highly accurate resolution even without taking very small time steps.

In the simulation domain protons are uniformly distributed, hence the number density of particles is one per cell. The initial velocity distribution function for protons is Maxwellian with the thermal velocity $v_{\text {th }}=0.1 V_{\mathrm{A}}$. The simulation time step is $\omega_{\text {ci }} \delta t=0.05$. The boundary conditions for protons are open in all three directions.

\section{A model of a single-loop flare}

In this section we briefly summarize the concept of a "moving solitary magnetic kink" (MoSMaK) that was found from 3-D MHD simulation (Sakai et al. 2002). We investigate head-on collision process of two plasma blobs in a magnetic flux tube along the $y$-axis. The axial current strength along the magnetic flux tube is characterized by a parameter $q$ called as twist parameter.

The MoSMaK can be excited near the interface of the collision of two plasma blobs in a magnetic flux tube with axial current $(q \geq 0.7)$, while in a magnetic flux tube with very weak axial current like $q=0.1$ another nonlinear elementary excitation called as a "moving solitary magnetic sausage" (MoSMaS) (Sakai et al. 2003) can be excited. The MoSMaK is characterized as an isolated magnetic flux (toroidal magnetic flux) with a ring structure, accompanying a pair of counter-rotating vortex rings. We find a standing solitary magnetic kink that appears near the interface of two plasma blobs when the two plasma blobs collide with the same velocity. We investigated the rear-end collision process of two plasma blobs where one plasma blobs remains at rest. We also found the formation of the MoSMaK. Therefore, the concept of the MoSMaK is important for understanding the dynamics of magnetic flux tubes with non-stationary plasma flows frequently observed in the flux tubes of solar plasmas.

Next we investigate the collision process of two MoSMaKs propagating along a bent magnetic flux tube (Sakai et al. 2002). Sakai et al. (2002) showed that during the collision process of two MoSMaKs about $20 \%$ of the magnetic field energy can be converted to the plasma heating as well as the expanding plasma kinetic energy. In Fig. 1a we show a schematic picture of a single loop flare that is triggered during collision of two moving solitary magnetic kinks (MoSMaKs) excited at the collision fronts between upflows and loop plasma. In Fig. 1b we show the simulation region and coordinate system.

In this section we present the simulation results of two cases of the plasma beta in the center of the loop: one is $\beta=0.5$ and the other case is $\beta=0.05$ to obtain the electromagnetic fields by using the resistive MHD equations. Here we take a long system size in the $z$-direction as $N_{z}=300$ and $N_{x}=N_{y}=200$, where $N_{x}, N_{y}$ and $N_{z}$ are the number of grid 


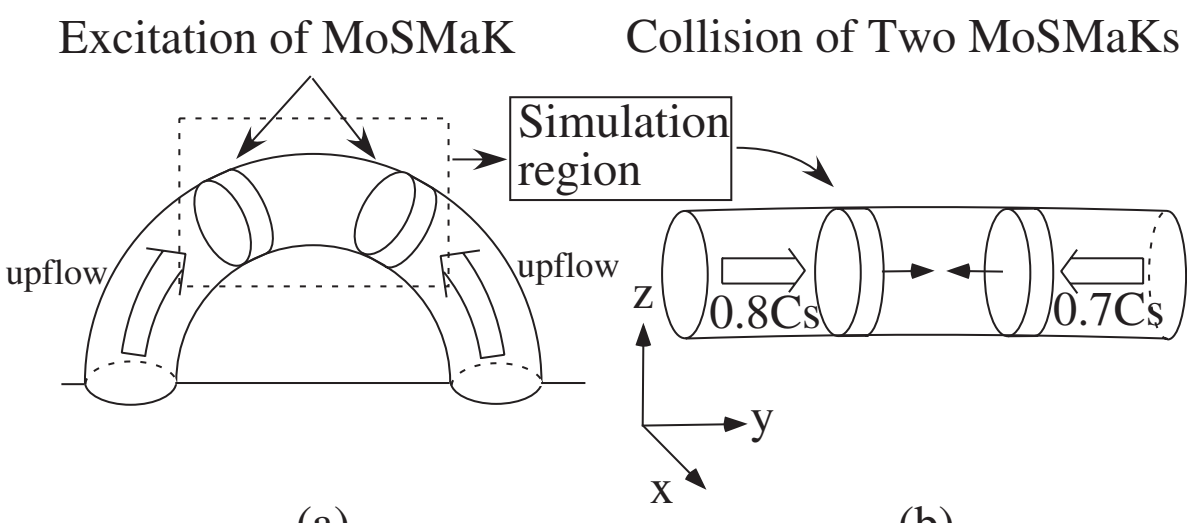

(a)

(b)

Fig. 1. a) Schematic picture of a single loop flare that is triggered during collision of two moving solitary magnetic kinks (MoSMaKs) excited at the collision fronts between upflows and loop plasma. b) Simulation region and coordinate system.

points in the $x$-, $y$ - and $z$-direction, respectively. The magnetic flux tube is assumed to be in an equilibrium state $(\nabla p=\boldsymbol{J} \times$ $\boldsymbol{B} / c)$, in which the magnetic field and pressure are given as

$B_{x}=q B_{y}\left(z-z_{\mathrm{c}}\right) / a$,

$B_{y}=-B_{0} \mathrm{e}^{-\left(\frac{r}{a}\right)^{2}}$,

$B_{z}=-q B_{y}\left(x-x_{\mathrm{c}}\right) / a$,

$p=\left(\frac{q^{2}}{2}-\frac{q^{2} r^{2}}{a^{2}}-1\right) \mathrm{e}^{-2\left(\frac{r}{a}\right)^{2}}+p_{00}$,

where $r=\sqrt{\left(x-x_{\mathrm{c}}\right)^{2}+\left(z-z_{\mathrm{c}}\right)^{2}}$. The center of the flux tube is placed at $\left(x_{\mathrm{c}}, z_{\mathrm{c}}\right)=(100,100)$ and the radius is $a=20$. The twist parameter $q$ is set to 1.0 and for $B_{0}=1.0$. $p_{00}$ is taken as $p_{00}=1.0$ for $\beta=0.5$ and $p_{00}=0.55$ for $\beta=0.05$, respectively. The axial current flows in the positive $y$-direction. The two MoSMaKs are excited due to rear-end collision near at $y=50$ and $y=150$, where the plasma in the flux tube between $y=70$ and $y=130$ is initially assumed at rest. We also take into account the density gradient in the $z$-direction. To consider a slightly bent magnetic flux tube, we impose the up-flow in the $z$-direction. The density and velocity associated with two plasma blobs are given as follows,

$$
\begin{aligned}
\rho= & p+\left\{0.5 \rho_{\mathrm{c}}\left(1-\tanh \left(\frac{y-y_{1}}{3}\right)\right)\right. \\
& \left.+0.5 \rho_{\mathrm{c}}\left(1+\tanh \left(\frac{y-y_{2}}{3}\right)\right)\right\} \mathrm{e}^{-\left(\frac{r}{10}\right)^{2}+50 \mathrm{e}^{-\frac{z}{100}},}
\end{aligned}
$$

$V_{x}=0$,

$$
\begin{aligned}
V_{y}= & \left\{0.4 V_{y 1}\left(1-\tanh \left(\frac{y-y_{1}}{3}\right)\right)\right. \\
& \left.-0.5 V_{y 2}\left(1+\tanh \left(\frac{y-y_{2}}{3}\right)\right)\right\} \mathrm{e}^{-\left(\frac{r}{10}\right)^{2},}
\end{aligned}
$$

$V_{z}=0.4 \mathrm{e}^{-\left(\frac{r}{20}\right)^{2}} \mathrm{e}^{-\left(\frac{y-100}{100}\right)^{4}}$,

where $y_{1}=50, y_{2}=150, \rho_{\mathrm{c}}=10$, and $V_{y 1}=0.8 C_{\mathrm{s}}$, and $V_{y 2}=0.7 C_{\mathrm{s}}$.

In Fig. 2 we present the time evolution of the isosurface of total magnetic field intensity with $|\boldsymbol{B}|=0.3$ during collision (a)
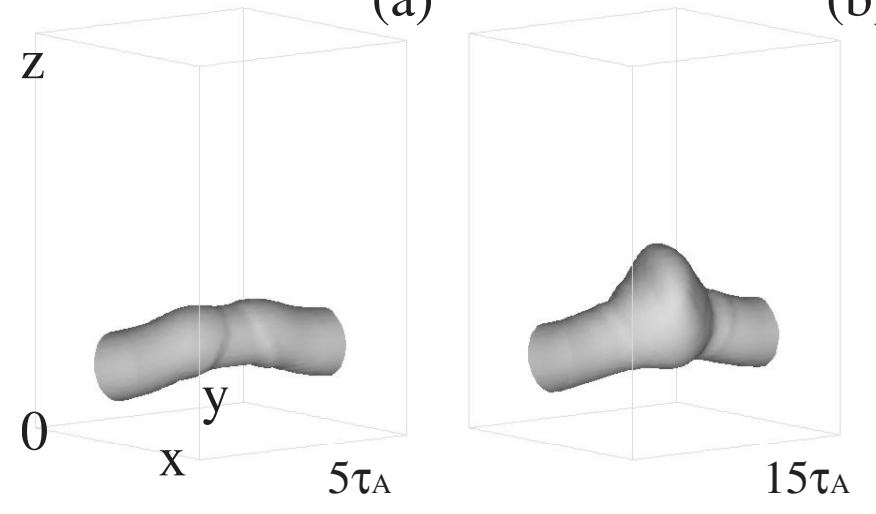

(c)
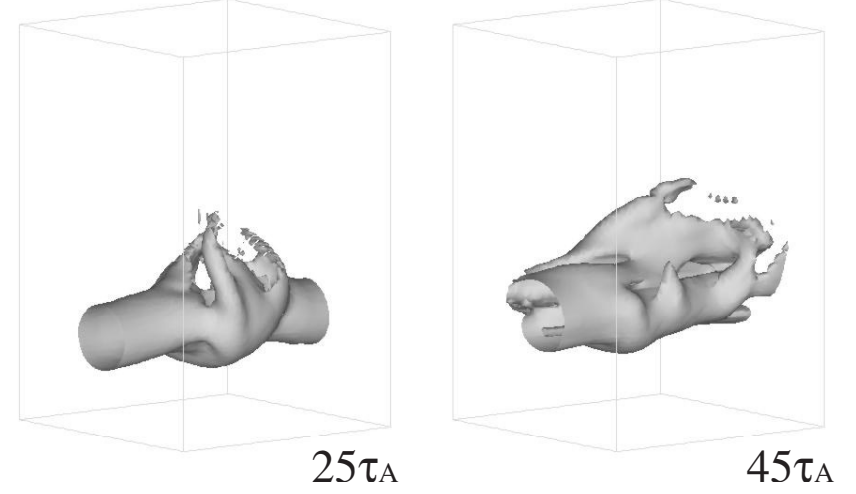

Fig. 2. The time evolution of the isosurface of total magnetic field intensity with $|\boldsymbol{B}|=0.3$ during collision of two MoSMaKs for $\beta=0.5$ at four different times: a) just before the collision, b) just after the collision, c) and d) disruption of a loop.

of two MoSMaKs for $\beta=0.5$ at four different times: (a) at $5 \tau_{\mathrm{A}}$ (just before the collision), (b) at $15 \tau_{\mathrm{A}}$ (just after the collision), (c) at $25 \tau_{\mathrm{A}}$ and (d) at $45 \tau_{\mathrm{A}}$ (disruption phase of a loop). In Fig. 3 we present the time evolution of the isosurface of total magnetic field intensity with $|\boldsymbol{B}|=0.3$ during collision of two MoSMaKs for $\beta=0.05$ at four different times: (a) at $5 \tau_{\mathrm{A}}$ (just before the collision), (b) at $15 \tau_{\mathrm{A}}$ (just after the collision), (c) at $25 \tau_{\mathrm{A}}$ and (d) at $45 \tau_{\mathrm{A}}$ (disruption phase of a loop). 
(a)
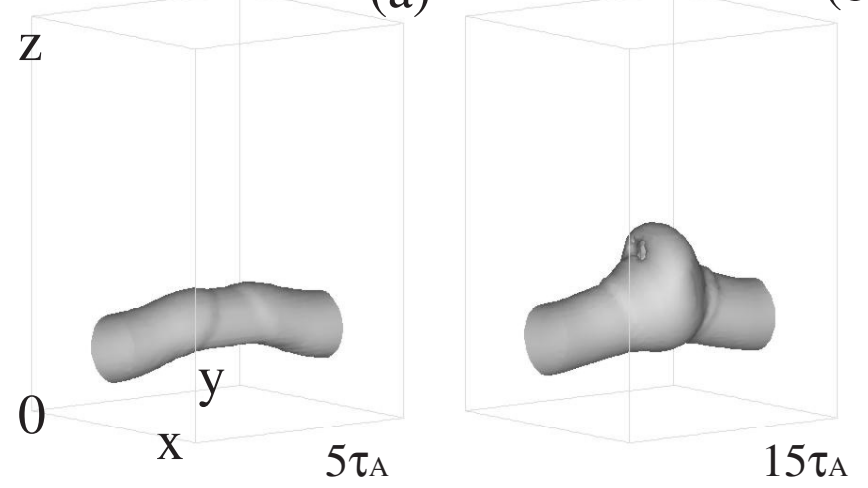

(c)

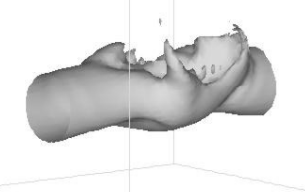

$25 \tau_{\mathrm{A}}$

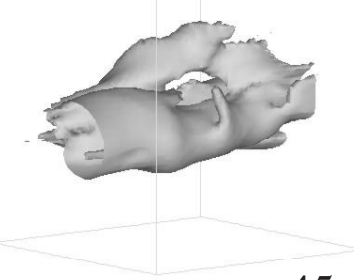

$45 \tau_{\mathrm{A}}$ (b)
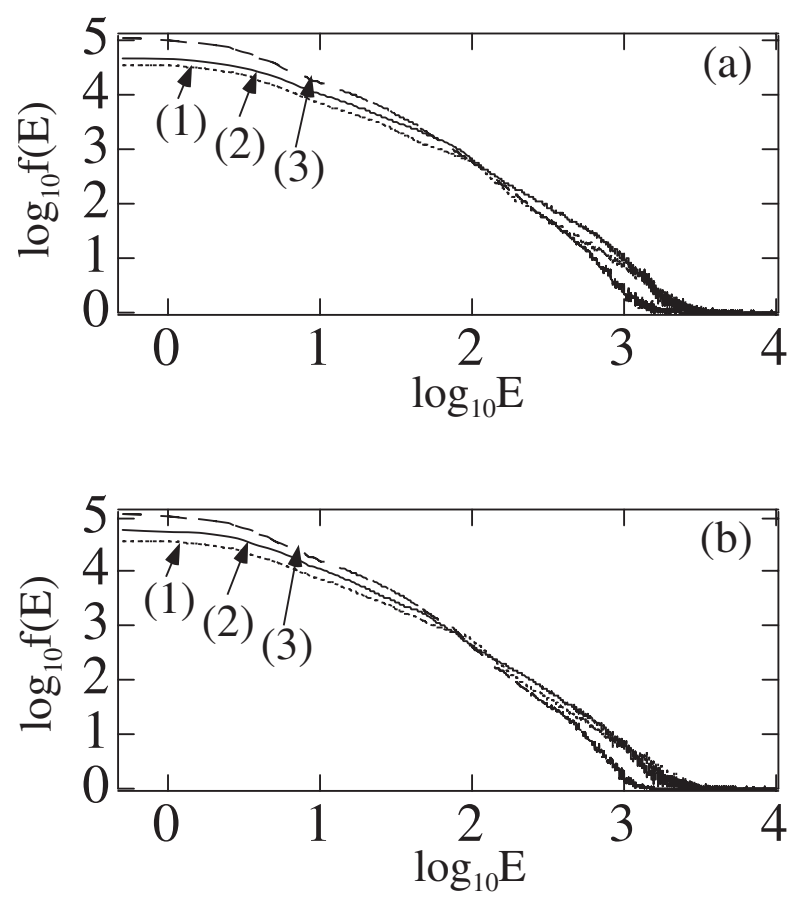

Fig. 4. a) Proton energy spectra at three different times, at (1) $15 \tau_{\mathrm{A}}$ (dotted line), (2) $25 \tau_{\mathrm{A}}$ (solid line) and (3) $45 \tau_{\mathrm{A}}$ (dashed line) for $\beta=0.5$. b) Proton energy spectra at three different times, at (1) $15 \tau_{\mathrm{A}}$ (dotted line), (2) $25 \tau_{\mathrm{A}}$ (solid line) and (3) $45 \tau_{\mathrm{A}}$ (dashed line) for $\beta=0.05 . E=\left(V_{x}^{2}+V_{y}^{2}+V_{z}^{2}\right) / V_{\mathrm{A}}^{2}$.

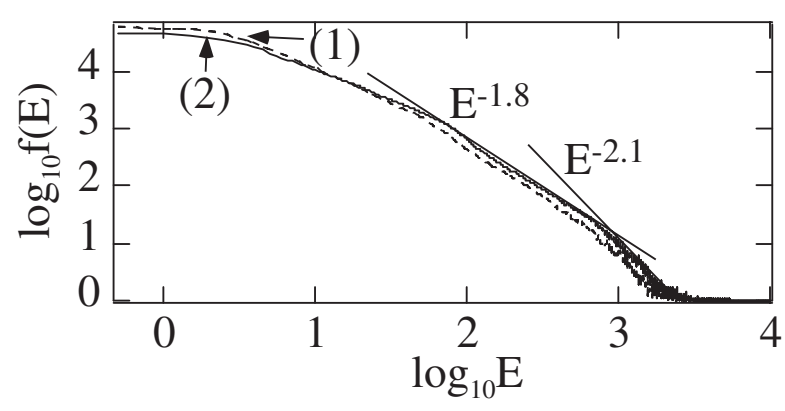

Fig. 5. Proton energy spectra at $\omega_{\mathrm{ci}} t=1500$, showing double power law whose index is about 1.8 and 2.1 (1) for $\beta=0.05$ (dotted line) and (2) for $\beta=0.5$ (solid line).

double power law whose index is about 1.8 and 2.1 for both cases of $\beta=0.05$ (dotted line) and $\beta=0.5$ (solid line). Since the aim of this study is to investigate the acceleration of protons in a single-loop solar flare, we establish physical links between the scaling of the basic parameters in the simulations and solar flare conditions. As the initial given value of $A=$ $V_{\mathrm{A}} / c=1 / 300$, the Alfvén velocity is about $1000 \mathrm{~km} \mathrm{~s}^{-1}$. Also, the proton thermal velocity is assumed to be equal to $0.1 V_{\mathrm{A}}$, or $100 \mathrm{~km} \mathrm{~s}^{-1}$, which corresponds to the $\sim 100 \mathrm{eV}$ ambient protons. The maximum proton energy is about $25 \mathrm{MeV}$. There appears a breaking point of the index from 1.8 to 2.1 near $10 \mathrm{MeV}$. The acceleration time $\omega_{\mathrm{ci}} t=1500$ of the protons is about $10^{-3} \mathrm{~s}$ and quite rapid compared with the MHD time scale $\tau_{\mathrm{A}}=a / V_{\mathrm{A}}=10 \mathrm{~s}$, if we take an ion-cyclotron frequency of $1.34 \times 10^{6} \mathrm{rad} / \mathrm{s}$ for a magnetic field of $140 \mathrm{G}$ and $a=10^{9} \mathrm{~cm}$. 
Next we investigate the mechanism of the protons for the case of $\beta=0.05$. In Fig. 6 we present the proton velocity distribution functions of three directions for $\beta=0.05$. The dotted curves show at $\omega_{\mathrm{ci}} t=0$, while the solid curves show at $\omega_{\mathrm{ci}} t=1500$. As seen in Figs. 6a and 6c, the velocity distributions are almost isotropic, while in the $y$-direction, as seen in Fig. $6 \mathrm{~b}$ the velocity distribution is asymmetric and the protons are accelerated mainly in positive $y$-direction. To understand the reason why the protons are accelerated in positive $y$-direction, in Fig. 7 we show the spatial distributions (gray scale) in the $x-z$ plane on $y=70$ of three components of magnetic field; $B_{x}$ (Fig. 7a), $B_{y}$ (Fig. 7b) and $B_{z}$ (Fig. 7c at $25 \tau_{\mathrm{A}}$ for $\beta=0.05$. The contour of the electric field intensity with $E_{y}=0.05$ is overplotted on the above figures. The region where the electric field $E_{y}$ is strong coincides with the region where the magnetic field $B_{y}$ is oriented in negative $y$ direction as seen in Fig. 7b. As seen in Figs. 7a and 7b, the region with strong $E_{y}$ coincides with the region where both $B_{x}$ and $B_{z}$ reverse their sign. This means that the strong $E_{y}$ region corresponds to the region where the current $J_{y}$ is localized and the magnetic reconnection occurs. From the electric and magnetic field data near the region where the current $J_{y}$ is formed, we can calculate the angle between the magnetic field line and electric field $E_{y}$ as $\theta=\cos ^{-1}\left(\left(\boldsymbol{E}_{y} \cdot \boldsymbol{B}\right) /\left|E_{y} \| \boldsymbol{B}\right|\right)$, that gives $\theta=14^{\circ}$. Therefore we conclude that the magnetic field lines are almost parallel to the electric field $E_{y}$ in this region.

In order to confirm the region of the proton acceleration by the electric field $E_{y}$, in Fig. 8a we present the spatial distribution of the electric field $E_{y}$ in the $x-z$ plane on $y=70$ at $25 \tau_{\mathrm{A}}$ and in Fig. $8 \mathrm{~b}$ we present the spatial distribution of the electric field $E_{y}$ in the $y-z$ plane on $x=100$ at $25 \tau_{\mathrm{A}}$ for $\beta=0.05$. The regions of dotted circle show that the electric field $E_{y}$ is strongest. In Fig. 8c the phase diagram of protons in the $x-V_{y}$ plane at $\omega_{\mathrm{ci}} t=1500$ is shown, while in Fig. $8 \mathrm{~d}$ the phase diagram of protons in the $y-V_{y}$ plane at $\omega_{\mathrm{ci}} t=1500$ is shown. From the comparison between Figs. $8 \mathrm{a}$ and $8 \mathrm{c}$, we find that the protons located near $x=100$ can be effectively accelerated to positive $y$-direction due to the strong electric field $E_{y}$. This is the reason that as seen in Fig. 6b, the velocity distribution in the $y$-direction is asymmetric and the protons are accelerated mainly in positive $y$-direction. From Fig. 8d, we find that there exists another region that the protons can be effectively accelerated near $y=140$ along the loop where the electric field $E_{y}$ is strong in the positive y-direction as seen in Fig. 8b. Therefore we conclude that the high-energy protons for the case of $\beta=0.05$ can be produced mainly by locally strong electric field $E_{y}$ along the loop that is generated associated with the local magnetic reconnection process.

We investigate the proton acceleration for the case of $\beta=$ 0.5 , by comparing the case of $\beta=0.05$. In Figs. $9 \mathrm{a}$ and $9 \mathrm{~b}$ we show the phase diagram of protons in the $z-V_{y}$ plane at $\omega_{\mathrm{ci}} t=$ 1500 for $\beta=0.05$ and for $\beta=0.5$, respectively. The region of dot-dashed circle in Fig. $9 \mathrm{~b}$ shows that protons located around $z=130$ near the upper part of the disrupted loop are accelerated mainly in the positive $y$-direction up to $V_{y}=25 V_{\mathrm{A}}$ only for $\beta=0.5$. To understand the proton acceleration in the upper part for $\beta=0.5$, in Figs. $9 \mathrm{c}$ and $9 \mathrm{~d}$ we show the spatial distribution of the electric field $E_{y}$ in the $x-y$ plane on $z=130$ at $25 \tau_{\mathrm{A}}$
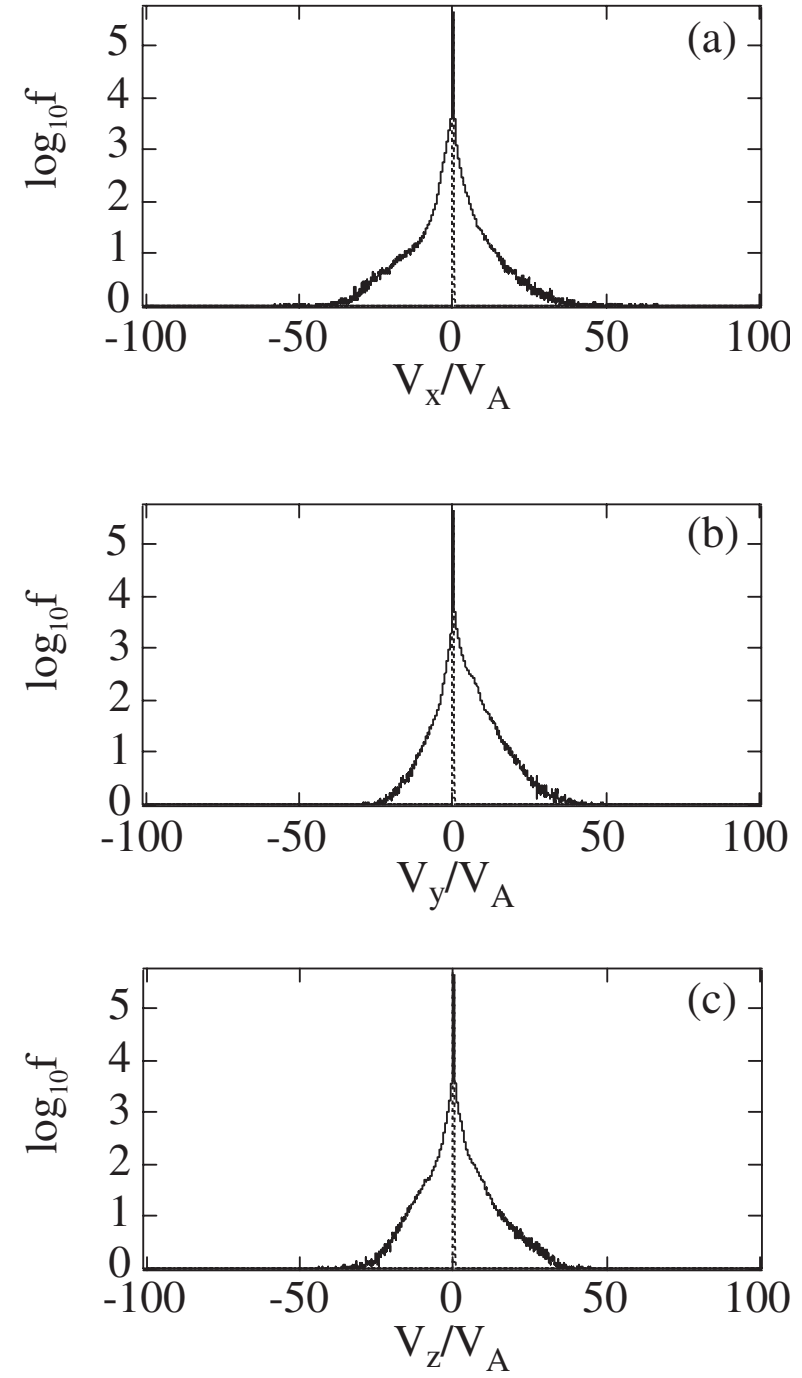

Fig. 6. Proton velocity distribution functions of a) the $x$-direction, b) $y$-direction, and c) $z$-direction for $\beta=0.05$. Dotted curves show at $\omega_{\mathrm{ci}} t=0$, while solid curves show at $\omega_{\mathrm{ci}} t=1500$.

for $\beta=0.05$ and $\beta=0.5$, respectively. In Figs. 9e and 9f, we also show the phase diagram of protons in the $x-V_{y}$ plane at $\omega_{\mathrm{ci}} t=1500$ for $\beta=0.05$ and for $\beta=0.5$, respectively. The solid circles and dotted circles in Figs. 9d and 9f correspond each other. From the comparison of these regions we conclude that the protons located near the upper part of the disrupted loop for $\beta=0.5$ can be effectively accelerated due to locally strong electric field $E_{y}$. The reason why the elecctric field near the upper part of the disrupted loop becomes strong is due to the fact that the loop with $\beta=0.5$ can be strongly deformed to the upper part as well as inside the loop, therefore there occur current sheets where magnetic reconnection is possible.

Finally we present simulation results of two cases with the symmetric collision velocity $\left(0.7 C_{\mathrm{s}}\right)$ and with the collision cross-section smaller than the present case, to see how the final proton acceleration is affected by the colliding states. In Fig. 10 we show the comparison of the proton energy spectrum (a) and three components (b)-(d) of the proton velocity distribution functions at $\omega_{\mathrm{ci}} t=1500$ between (1) solid lines: the present case with unsymmetric collision velocities 
(a)

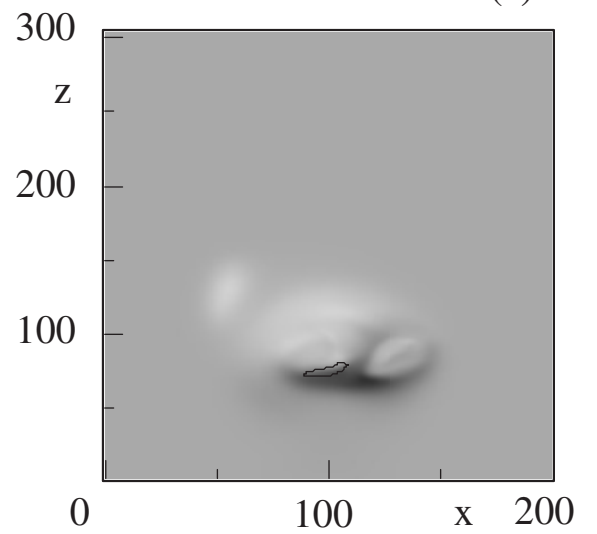

(b)

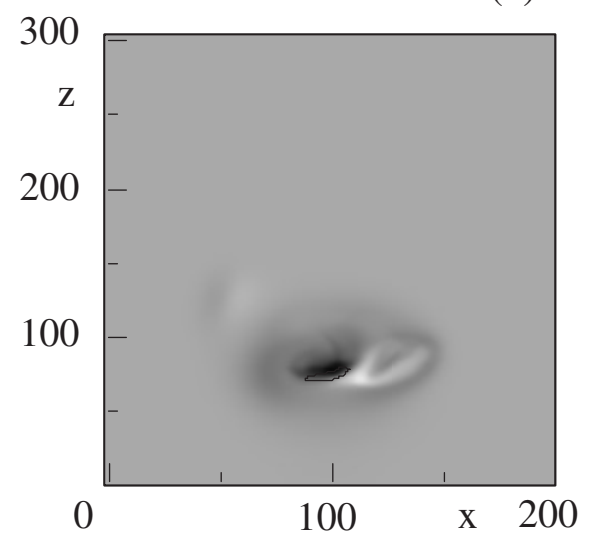

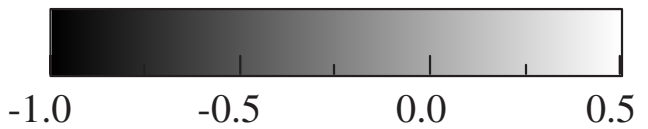

(c)

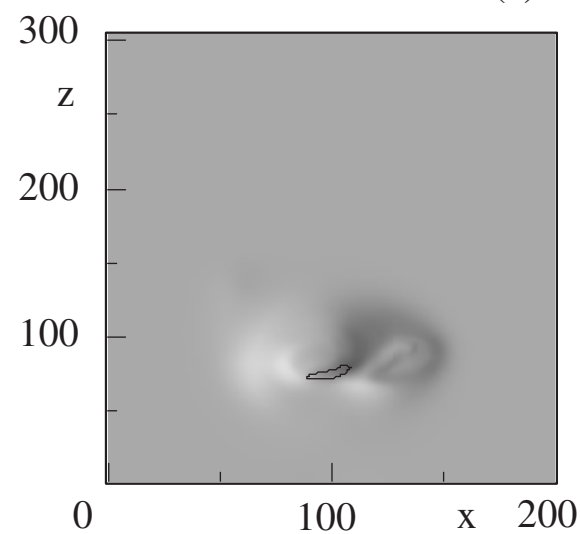

Fig. 7. Spatial distributions (gray scale) in the $x-z$ plane on $y=70$ of three components of magnetic field a) $B_{x}$. b) $B_{y}$ and c) $B_{z}$ at $25 \tau_{\mathrm{A}}$ for $\beta=0.05$. The contour of the electric field intensity with $E_{y}=0.05$ is overplotted.

of $0.7 C_{\mathrm{s}}$ and $0.8 C_{\mathrm{s}}$, (2) dotted lines: symmetric collision velocity of $0.7 C_{\mathrm{s}}$ and (3) dashed lines: unsymmetric collision velocities of $0.7 C_{\mathrm{s}}$ and $0.8 C_{\mathrm{s}}$ and with the collision cross-section smaller than the cases of (1) and (2). The collision cross-secton of the case (3) that the centers of colliding clouds are located at $x=105, z=50$ for the cloud with $0.8 C_{\mathrm{s}}$ and at $x=95, z=50$ for the cloud with $0.7 C_{\mathrm{s}}$ becomes smaller with about a half cross-section than the present case. As seen in Fig. 10a, the proton energy spectrum are almost same for symmetric and unsymmetric cloud collision. As the collision cross-section becomes smaller for the case (3), the number of accelerated protons becomes slightly decreased compared with other two cases, as seen in Figs. 10a and 10d. As seen in Fig. 10c, the protons are accelerated dominantly in the positive $y$-direction for three cases. Therefore we conclude that the one-direction acceleration along the flux tube for protons is not affected by above collision parameters such as the collision velocity and the collision cross-section.

\section{Discussion and conclusions}

We investigated a new model of single-loop flare based on the fact that a magnetic flux tube with axial current can be explosively disrupted during collision of two moving solitary magnetic kinks (MoSMaK), particularly paying attention to an acceleration mechanism of high energy protons. By using three-dimensional electromagnetic fields obtained from a resistive three-dimensional MHD equations during the single-loop flare, we investigated the orbit of many protons to obtain their energy spectra. We found that the protons can be accelerated to $\gamma$-ray-emitting energies $(>1 \mathrm{MeV}$ ) with double power-law spectra up to about $25 \mathrm{MeV}$. There appears a breaking point of the index of the power-law spectra from 1.8 to 2.1 near $10 \mathrm{MeV}$. The acceleration time $\omega_{\mathrm{ci}} t=1500$ of the protons is about $10^{-3} \mathrm{~s}$ and quite rapid compared with the MHD time scale $\tau_{\mathrm{A}}=a / V_{\mathrm{A}}=10 \mathrm{~s}$, if we take an ion-cyclotron frequency of $1.34 \times 10^{6} \mathrm{rad} / \mathrm{s}$ for a magnetic field of $140 \mathrm{G}$ and 
(a)

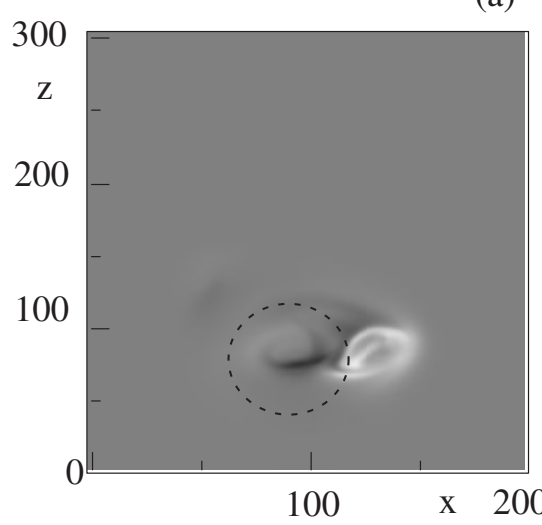

(b)

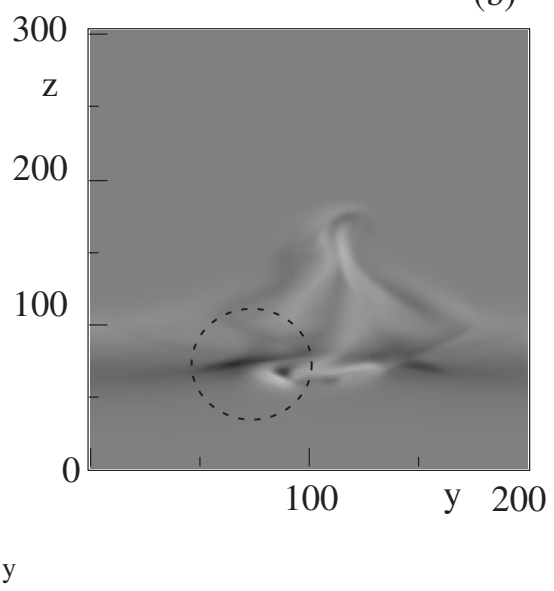

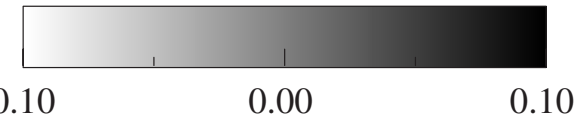

(c)

(d)
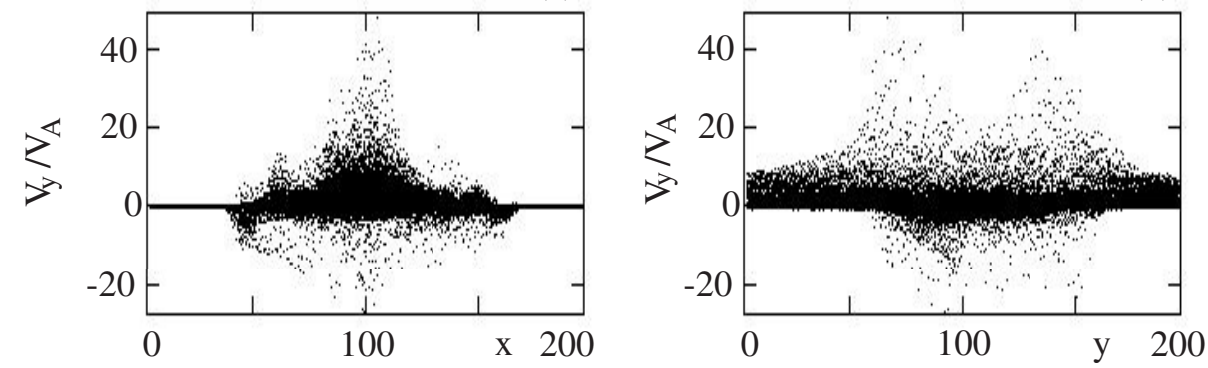

Fig. 8. a) Spatial distribution of the electric field $E_{y}$ in the $x-z$ plane on $y=70$ at $25 \tau_{\mathrm{A}}$ for $\beta=0.05$. b) Spatial distribution of the electric field $E_{y}$ in the $y-z$ plane on $x=100$ at $25 \tau_{\mathrm{A}}$ for $\beta=0.05$. The regions of dotted circle show that the electric field $E_{y}$ is strongest. c) The phase diagram of protons in the $x-V_{y}$ plane at $\omega_{\mathrm{ci}} t=1500$. d) The phase diagram of protons in the $y-V_{y}$ plane at $\omega_{\mathrm{ci}} t=1500$.

$a=10^{9} \mathrm{~cm}$. The protons are accelerated mainly in one direction along the loop by the electric field produced near the threedimensional localized current associated with the magnetic reconnection process in the disrupted loop. We studied the loop disruption for two different plasma beta in the center of the loop; $\beta=0.5$ and $\beta=0.05$. In a high beta case $(\beta=0.5)$, the disruption of the loop is more violent than that of $\beta=0.05$. We found that the proton acceleration for the case of $\beta=0.5 \mathrm{oc}-$ curs mainly at two different regions; one is inside the loop and the other is in the upper part of the disrupting loop. However, the energy spectra of protons are very similar for both cases.

As shown by Hurford et al. (2003) from the first gamma-ray images of a solar flare taken from the Reuven Ramaty High Energy Solar Spectroscopic Imager (RHESSI) for the X4.8 flare of 2002 July 23, the fact that protonassociated gamma-ray source does not coincide with the electron-bremsstrahlung sources suggests that the protons would be accelerated in one direction by the DC electric field and could subsequently interact in spatially separated sources. One of possible scenario to explain the above recent observation is as follows. The single loop with high-beta like $\beta=0.5$ in the center of the loop is supposed to be disrupted by the present mechanism, then the disrupted part of the loop with high energy protons as well as hot thermalized protons could move up and then interact with overlying other loop or magnetic arcade structure. The interaction between the ascending magnetized plasma blobs and other loop can lead to magnetic reconnection in the interaction region as discussed by Sakai et al. (1995), where the proton could be accelerated more by the inductive electric field associated with the magnetic reconnection mostly in one direction along the guiding magnetic field.

We compare our results with some observations of solar flares. Van Hollebeke et al. (1975) reported proton spectra, summarizing 185 solar proton events observed from IMP-4 and IMP-5 experimental data. They showed that these proton spectra approximately follow a power-law function in the $20-80 \mathrm{MeV}$ range. The power-law index varies with time during a flare. The spectra also vary from flare to flare. Flares located away from the magnetically connecting the Earth and the Sun exhibit steeper spectra. For flares located at helio-longitude W 20-80, the power-law index determined at the time of maximum particle intensity is thought to be representative of the source spectra. The power-law index is modified by the propagation effect at other times. For 32 flares located at W 20-80, the power-law index in the $20-80 \mathrm{MeV}$ range was shown by them. They gave seven events $(7 / 32=22 \%)$ showing the 
(a)

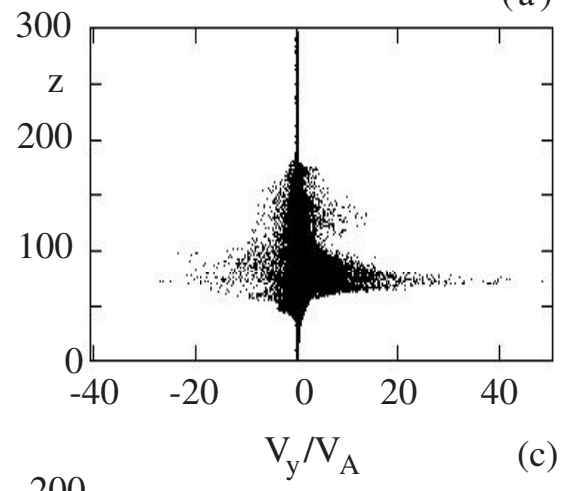

200

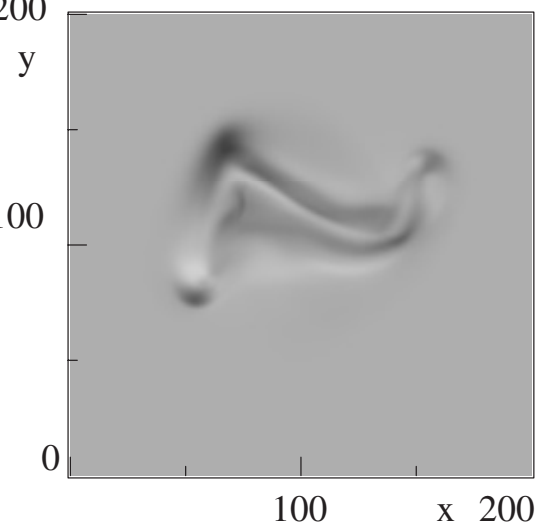

c)
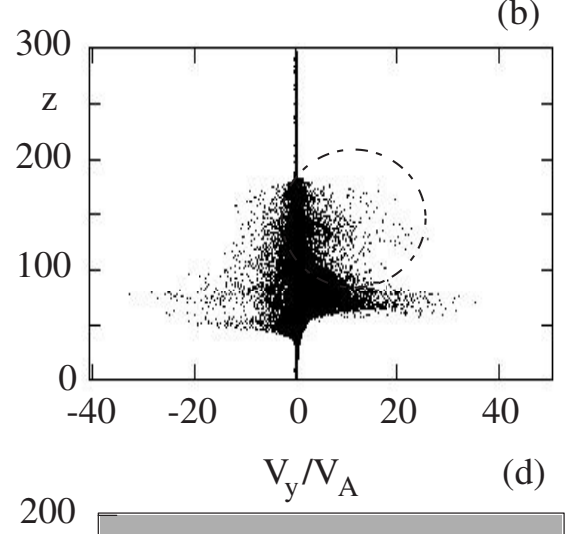

100

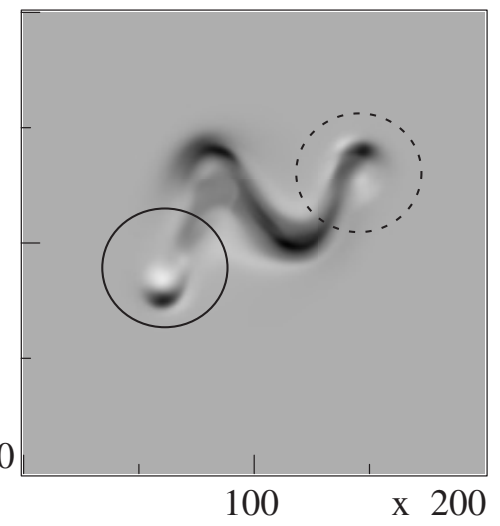

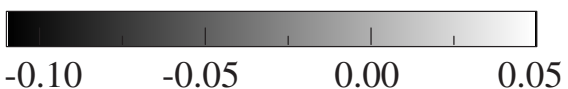

(e)
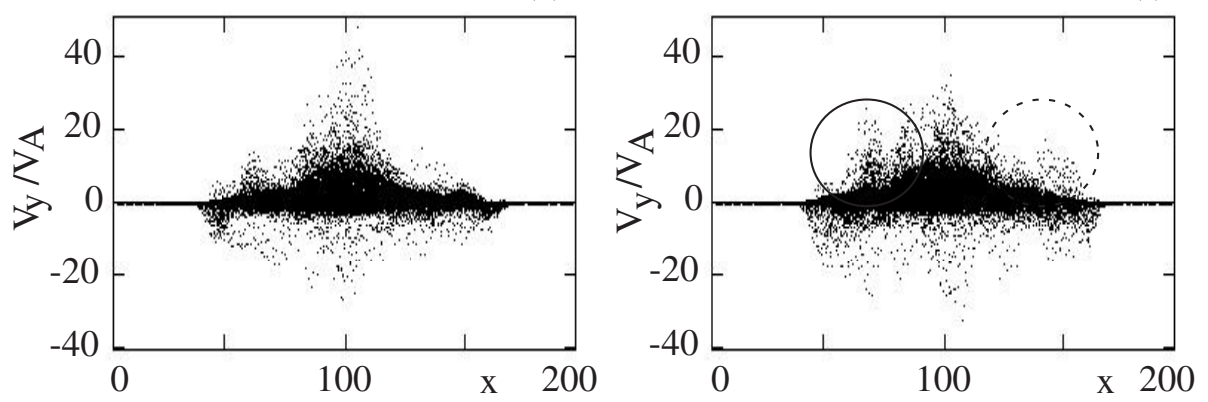

Fig. 9. a) The phase diagram of protons in the $z-V_{y}$ plane for $\beta=0.05$ at $\omega_{\mathrm{ci}} t=1500$. b) The phase diagram of protons in the $z-V_{y}$ plane for $\beta=0.5$ at $\omega_{\text {ci }} t=1500$. The region of dot-dashed circle shows that protons located near upper part of disrupted loop are accelerated for $\beta=0.5$. c) Spatial distribution of the electric field $E_{y}$ in the $x-y$ plane on $z=130$ at $25 \tau_{\mathrm{A}}$ for $\beta=0.05$. d) Spatial distribution of the electric field $E_{y}$ in the $x-y$ plane on $z=130$ at $25 \tau_{\mathrm{A}}$ for $\beta=0.5$. e) The phase diagram of protons in the $x-V_{y}$ plane for $\beta=0.05$ at $\omega_{\mathrm{ci}} t=1500$. f) The phase diagram of protons in the $x-V_{y}$ plane for $\beta=0.5$ at $\omega_{\mathrm{ci}} t=1500$. Solid circles and dotted circles in d) and f) correspond each other.

spectral index of 1.8-2.2, which agrees with the present simulation result. Other events $(78 \%)$ have the index in the range of 2.2-3.2, in which nine events show the index of 2.6-2.8.

Proton spectra in the energy range of $10-100 \mathrm{MeV}$ can be derived from the ratio between $2.22 \mathrm{MeV}$ (neutron capture line) to $\mathrm{C}$ and $\mathrm{O}$ de-excitation line fluorescence, which is the time-integrated flux. Ramaty et al. (1993) calculated the proton spectra using this method. Their conclusion is that most of the spectral indices are in the range of 3.0-4.6, which are harder than the present simulation result. Therefore we still need further investigations to understand fully the proton acceleration mechanism(s) in the impulsive phase of solar flares.
Acknowledgements. We thank the referee for useful comments. This work was finished when one of authors (J. I. Sakai) stayed in Warwick University, supported by the exchange program between JSPS and Royal Society. He thanks V. Nakariakov for his hospitality.

\section{References}

Alfvén, H., \& Carlqvist, P. 1967, Sol. Phys., 1, 220

Aschwanden, M. J. 2002, Space Sci. Rev., 101, Nos. 1-2, 1

Birdsall, C. K., \& Langdon, A. B. 1991, Plasma Physics via Computer Simulation (Adam Hilger), 58

Boris, J. P. 1970, Proc. Fourth Conf. Num. Sim. Plasmas, Naval Res. Lab., Wash. D.C., 3-67, 2-3 November 

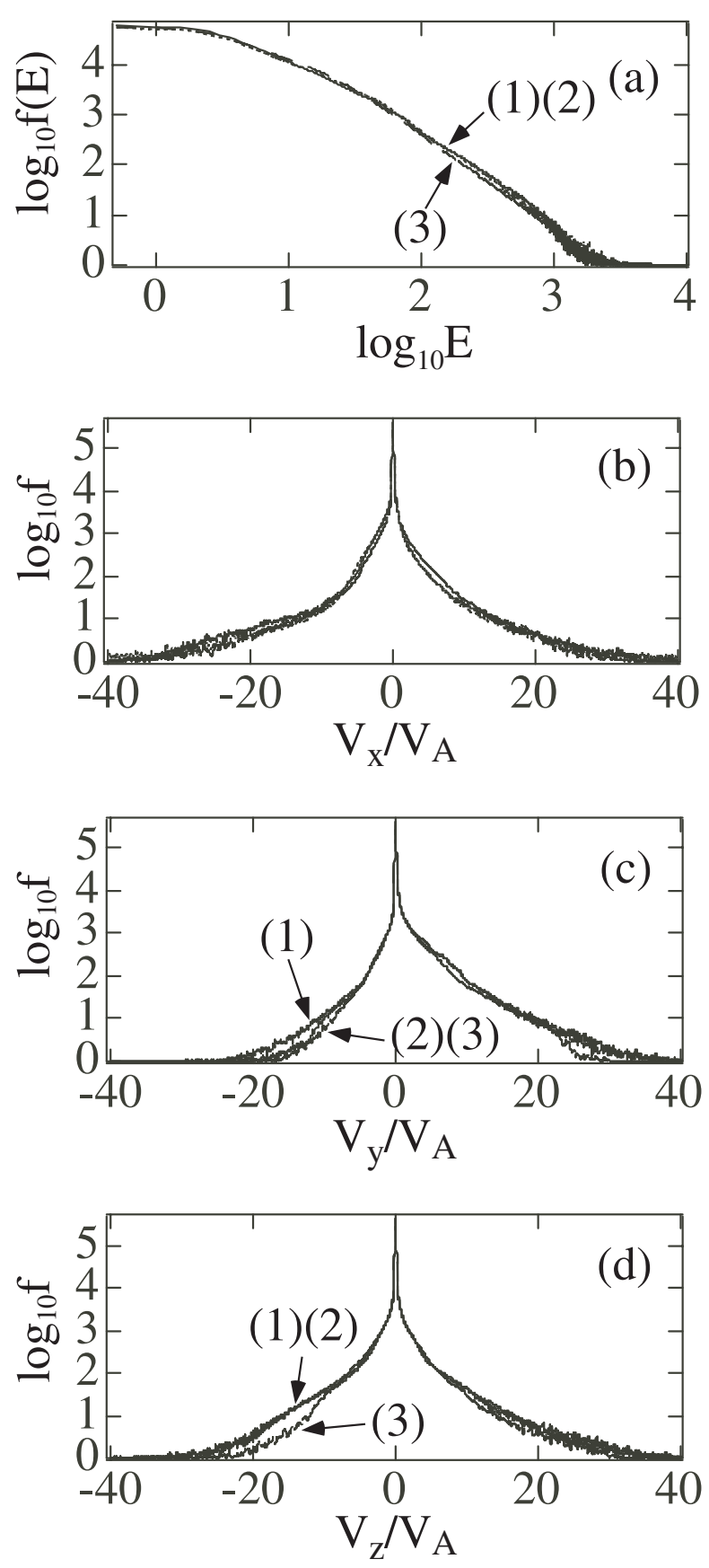

Fig. 10. Comparison of the proton energy spectrum a) and three components b)-d) of the proton velocity distribution functions at $\omega_{\mathrm{ci}} t=$ 1500 between (1) solid lines: the present case with unsymmetric collision velocities of $0.7 C_{\mathrm{s}}$ and $0.8 C_{\mathrm{s}},(2)$ dotted lines: symmetric collision velocity of $0.7 C_{\mathrm{s}}$ and (3) dashed lines: unsymmetric collision velocities of $0.7 C_{\mathrm{s}}$ and $0.8 C_{\mathrm{s}}$ and with the collision cross-section smaller than the cases of (1) and (2).

\section{Bulanov, S. V. 1980, SvA Lett., 6, 206}

Buneman, O. 1993, in Computer Space Plasma Physics, Simulation Techniques and Software, ed. H. Matsumoto, \& Y. Omura (Tokyo: Terra Scientific), 67
Chae, J., Qiu, J., Wang, H., \& Goode, P. 1999, ApJ, 513, L75

Chae, J., Wang, H., Goode, P., Fludra, A., \& Schuehle, U. 2000, ApJ, 528, L119

Colgate, S. A. 1978, ApJ, 221, 1068

Craig, I. J. D., \& Litvinenko, Yuri E. 2002, ApJ, 570, 387

Deeg, H. J., Borovsky, J. E., \& Duric, N. 1991, Phys. Fluids B3, 2660

De Jager, C. 1988, Proc. 20th Cosmic Ray Conf., Moscow 7, 66

De Jager, C., \& Sakai, J. I. 1991, Sol. Phys., 133, 395

Forman, M. A., Ramaty, R., \& Zweibel, E. G. 1986, in Physics of the Sun, ed. P. A. Sturrock, T. E. Holzer, D. M. Mihalas, \& R. K. Ulrich (D. Reidel Publishing Co), Chapter 13, 2

Forrest, D. J., \& Chupp, E. L. 1983, Nature, 305, 291

Furusawa, K., \& Sakai, J. I. 2000, ApJ, 540, 1156

Harra, L. K., Matthews, S. A., \& Culhane, J. L. 2001, ApJ, 549, L245

Hamilton, B., McClements, K. G., Fletcher, L., \& Thyagaraja, A. 2003, Sol. Phys., 214, 339

Heerikhuisen, J., Litvinenko, Yuri E., \& Craig, I. J. D. 2002, ApJ, 566, 512

Hurford, G. J., Schwartz, R. A., Krucker, S., et al. 2003, ApJ, 595, L77

Lee, C-Y., Chae, J., \& Wang, H. 2000, ApJ, 545, 1124

Miller, J. A., Cargill, P. J., Emslie, G., et al. 1997, J. Geophys. Res., 102/A7, 14631

Miyagoshi, T., Uchida, Y., Yabiku, T., Hirose, S., \& Cable, S. 2001, PASJ, 53, 341

Mori, K., Sakai, J. I., \& Zhao, J. 1998, ApJ, 494, 430

Nishikawa, K. I., Sakai, J. I., Zhao, J., Neubert, T., \& Buneman, O. 1994, ApJ, 434, 363

Nocera, L., Pegoraro, F., Bulanov, S. V., \& Bertin, G. 1996, Phys. Scr., T63, 197

Ramaty, R., Mandzhavidze, N., Kozlovsky, B., \& Skibo, J. G. 1993, Adv. Space Res., 13, No.9, 275

Sakai, J. I. 1990, ApJS, 73, 321

Sakai, J. I. 1992, Sol. Phys., 140, 99

Sakai, J. I., \& de Jager, C. 1989, Sol. Phys., 123, 389

Sakai, J. I., \& de Jager, C. 1991, Sol. Phys., 134, 329

Sakai, J. I., \& de Jager, C. 1996, Space Sci. Rev., 77, 1

Sakai, J. I., Fushiki, T., \& Nishikawa, K. I. 1995, Sol. Phys., 158, 301

Sakai, J. I., Kawata, T., Yoshida, K., Furusawa, K., \& Cramer, N. F. 2000, ApJ, 537, 1063

Sakai, J. I., Nishi, K., \& Sokolov, I. V. 2002, ApJ, 576, 519

Sakai, J. I., Nishi, K., \& Sokolov, I. V. 2003, ApJ, 584, 1095

Sakai, J. I., \& Ohsawa, Y. 1987, Space Sci. Rev., 46, 113

Simnett, G. M. 1995, Space Sci. Rev., 73, 387

Sokolov, I. V., Timofeev, E. V., Sakai, J. I., \& Takayama, K. 1999, Shock waves, 9,423

Spicer, D. F. 1977, Sol. Phys., 53, 305

Tajima, T., Brunel, F., \& Sakai, J. I. 1982, ApJ, 245, L45

Uchida, Y., Miyagoshi, T., Yabiku, T., Cable, S., \& Hirose, S. 2001, PASJ, 53, 331

Uchida, Y., \& Shibata, K. 1988, Sol. Phys., 116, 291

Van Hollebecke, M. A. I., MaSung, L. S., \& McDonald, F. B. 1975, Sol. Phys., 41, 189

Zaitsev, V. V., Urpo, S., \& Stepanov, A. V. 2000, A\&A, 357, 1105 